\title{
Regulation of RIG-I Activation by K63-Linked Polyubiquitination
}

\author{
Masaaki Okamoto', Takahisa Kouwaki', Yoshimi Fukushima ${ }^{1}$ and Hiroyuki Oshiumi',2* \\ ${ }^{1}$ Faculty of Life Sciences, Department of Immunology, Graduate School of Medical Sciences, Kumamoto University, \\ Kumamoto, Japan, ${ }^{2}$ PRESTO, Japan Science and Technology Agency, Kumamoto, Japan
}

OPEN ACCESS

Edited by: Uday Kishore, Brunel University London, United Kingdom

Reviewed by:

Taruna Madan, National Institute for Research in Reproductive Health, India Junji Xing,

Houston Methodist Research Institute, United States

*Correspondence: Hiroyuki Oshium oshiumi@kumamoto-u.ac.jp

Specialty section: This article was submitted to Molecular Innate Immunity, a section of the journal

Frontiers in Immunology

Received: 01 November 2017 Accepted: 18 December 2017 Published: 05 January 2018

Citation: Okamoto M, Kouwaki T, Fukushima Y and Oshiumi $H$ (2018) Regulation of RIG-I Activation by K63-Linked Polyubiquitination.

Front. Immunol. 8:1942. doi: 10.3389/fimmu.2017.01942
RIG-I is a pattern recognition receptor and recognizes cytoplasmic viral double-stranded RNA (dsRNA). Influenza A virus, hepatitis C virus, and several other pathogenic viruses are mainly recognized by $R \mid G-I$, resulting in the activation of the innate immune responses. The protein comprises $\mathrm{N}$-terminal two caspase activation and recruitment domains (2CARDs), an RNA helicase domain, and the C-terminal domain (CTD). The CTD recognizes 5 '-triphosphate viral dsRNA. After recognition of viral dsRNA, the protein harbors K63-linked polyubiquitination essential for RIG-I activation. First, it was reported that TRIM25 ubiquitin ligase delivered K63-linked polyubiquitin moiety to the 2CARDs. The polyubiquitin chain stabilizes a structure called the 2CARD tetramer, in which four 2CARDs assemble and make a core that promotes the aggregation of the mitochondrial antiviral-signaling (MAVS) protein on mitochondria. MAVS aggregation then triggers the signal to induce the innate immune responses. However, subsequent studies have reported that Riplet, MEX3C, and TRIM4 ubiquitin ligases are also involved in K63-linked polyubiquitination and the activation of RIG-I. MEX3C and TRIM4 mediate polyubiquitination of the 2CARDs. By contrast, Riplet ubiquitinates the CTD. The physiological significance of each ubiquitin ligases has been shown by knockout and knockdown studies, but there appears to be contradictory to evidence reported in the literature. In this review, we summarize recent findings related to K63-linked polyubiquitination and propose a model that could reconcile current contradictory theories. We also discuss the physiological significance of the ubiquitin ligases in the immune system against viral infection.

Keywords: RIG-I, ubiquitin, innate immunity, virus, signaling pathway

\section{INTRODUCTION}

Pattern recognition receptors (PRRs) recognize viral nucleic acids and trigger a signal to induce the innate immune responses during viral infection $(1,2)$. RIG-I is a cytoplasmic RNA helicase and a PRR that recognizes cytoplasmic $5^{\prime}$ tri- or diphosphate double-stranded RNA (dsRNA) (3-5). RIG-I binds relatively short dsRNA $(<1 \mathrm{kbp})$ and is involved in the recognition of various viral infections, such as influenza A and B viruses, Japanese encephalitis virus, hepatitis $\mathrm{C}$ virus (HCV), dengue virus, and West Nile virus (6-8). After recognition of viral RNA, RIG-I associates with an adaptor protein, mitochondrial antiviral-signaling (MAVS) protein, also called IPS-1, Cardif, and VISA (9-12), resulting in the aggregation of MAVS on the outer membrane of mitochondria. This 
aggregation triggers a signal to induce the expression of type I interferon (IFN) and other inflammatory cytokines (13).

The RIG-I protein comprises two caspase-activation and recruitment domains (2CARDs) at the $\mathrm{N}$-terminal region, an RNA helicase domain, and a C-terminal domain (CTD) (14-16). Viral dsRNA binds to the RNA helicase domain and the CTD, and $5^{\prime}$ tri- and diphosphate are recognized by the CTD $(16,17)$. The N-terminal 2CARDs are responsible for the association with MAVS and, therefore, are required for triggering downstream signaling (5). In resting cells, the C-terminal region, which includes the CTD and the linker region between the CTD and the helicase domain, suppresses the N-terminal 2CARDs $(14,18)$. Binding of the CTD to dsRNA induces the conformational change of the RIG-I protein, resulting in the release of the 2CARDs (14). Subsequently, the proteins assemble along viral dsRNA and form a nucleoprotein filament (19). The released 2CARDs also assemble and form a 2CARD tetramer structure (20). The structure functions as a core for MAVS aggregation on mitochondria (21).

The RIG-I protein harbors Lys 63-linked (K63-linked) polyubiquitination required for its activation (22). TRIM25 is a ubiquitin ligase and delivers K63-linked polyubiquitin moiety to the RIG-I 2CARDs $(22,23)$. The polyubiquitin chains stabilize the 2CARD tetramer structure (21). The physiological significance of TRIM25 in RIG-I activation has been shown by several studies (22-27). However, recent studies have reported three other ubiquitin ligases, RING finger protein leading to RIG-I activation (Riplet), mex-3 RNA-binding family member C (MEX3C), and TRIM4, which are required for the polyubiquitination and activation of RIG-I (28-30).

\section{POLYUBIQUITINATION OF THE 2CARDs OF RIG-I}

Ubiquitin ligases add a ubiquitin chain at $\mathrm{K}$ but not $\mathrm{R}$ residues of the target protein. There are $18 \mathrm{~K}$ residues in the RIG-I 2CARDs, and mass spectrometry analysis revealed that the 2CARDs fragment carried K63-linked polyubiquitin chains at K99, K169, K172, K181, K190, and K193 (22). Knockdown of TRIM25 abrogated polyubiquitination of the 2CARDs fragment, suggesting that TRIM25 mediates K63-linked polyubiquitination at the $6 \mathrm{~K}$ residues of the 2CARDs (22). The 2CARDs fragment has an ability to bind to MAVS, and overexpression of the 2CARDs fragment leads to auto-activation of signaling (5, 9-12). An amino acid substitution assay revealed that the substitution of $\mathrm{K} 172$, but not of other $\mathrm{K}$ residues, with $\mathrm{R}$ abrogated the signaling induced by the 2CARDs fragment (22). Knockout of TRIM25 severely reduced RIG-I-mediated type I IFN production during viral infection. These observations indicate the importance of TRIM25-mediated K172 ubiquitination (22).

Evidence also suggests that TRIM25 produces unanchored K63-linked polyubiquitin chains in response to viral infection and delivered them to RIG-I (23). The same study also showed that the K172 residue of RIG-I was important for non-covalent binding of RIG-I with unanchored polyubiquitin chains (23).
Considering that mass spectrometry analysis revealed the covalent binding of RIG-I with K63-linked polyubiquitin chains, these observations indicate that either covalent or non-covalent binding with polyubiquitin chains is sufficient for RIG-I 2CARDs activation $(23,26)$. A structural study of RIG-I 2CARDs tetramer provided evidence that both covalent and non-covalent binding of polyubiquitin chains promotes the formation of the 2CARD tetramer structure $(21,26)$. The TRIM25 activity itself is regulated by the physical interaction between the TRIM25 SPRY domain and RIG-I 2CARDs (31). The cooperative assembly of TRIM25 and RIG-I facilitates the dimerization of the TRIM25 RING domain, which is required for TRIM25 to make polyubiquitin chain (31).

An accumulating body of evidence has shown that TRIM25 delivers K63-linked polyubiquitin moiety to RIG-I 2CARDs for RIG-I activation and that the K172 residue is important for the binding of RIG-I to polyubiquitin chains $(26,27,32)$. However, subsequent studies revealed that not only K172 but also other $\mathrm{K}$ residues are also important for the binding of RIG-I to K63linked polyubiquitin chains. First, Shigemoto et al. reported that the expression of the RIG-I K172R full-length protein could compensate for a defect in RIG-I knockout mouse embryonic fibroblasts (MEFs) after Sendai virus infection (33). Second, two other ubiquitin ligases, MEX3C and TRIM4, were reported to mediate polyubiquitination of the RIG-I 2CARDs at other $\mathrm{K}$ residues $(29,30)$. Kuniyoshi et al. reported that MEX3Cmediated polyubiquitination was reduced by mutations at K48, K99, and K169, and Yan et al. reported that TRIM4 targeted K164 and K172 (30). Mass spectrometry analysis has also revealed the ubiquitination at K48, K96, K170, as well as K172 and K190 of the 2CARDs (34). They reported that simultaneous amino acids substitutions at K48, K96, and K172 substantially reduced the polyubiquitination of RIG-I (34). These observations suggest that K63-linked polyubiquitination at these $\mathrm{K}$ residues can compensate for the loss of K172 binding to the ubiquitin chain under certain conditions (Figure 1).

\section{POLYUBIQUITINATION OF THE RIG-I C-TERMINAL REGION}

Riplet, another ubiquitin ligase, is also involved in K63-linked polyubiquitination and activation of RIG-I. Riplet was first isolated by our yeast two-hybrid screening using the RIG-I CTD fragment as a bait (28). An immunoprecipitation assay then confirmed that the protein bound to the CTD fragment, and our studies also indicated that Riplet mediates K63-linked polyubiquitination of the RIG-I CTD (28). Our mutation analysis indicated that K849 and K851 of the CTD were important for Riplet-mediated polyubiquitination, and Riplet also targeted other $\mathrm{K}$ residues, including K888, K907, and K909 of the CTD and K788 in the linker region between the CTD and the helicase domain $(24,28)$ (Figure 1).

To assess the physiological significance of the protein, we generated Riplet knockout mice. Knockout of Riplet severely impaired the type I IFN and IL-6 production in MEF, macrophages, and conventional dendritic cells following influenza A virus and 


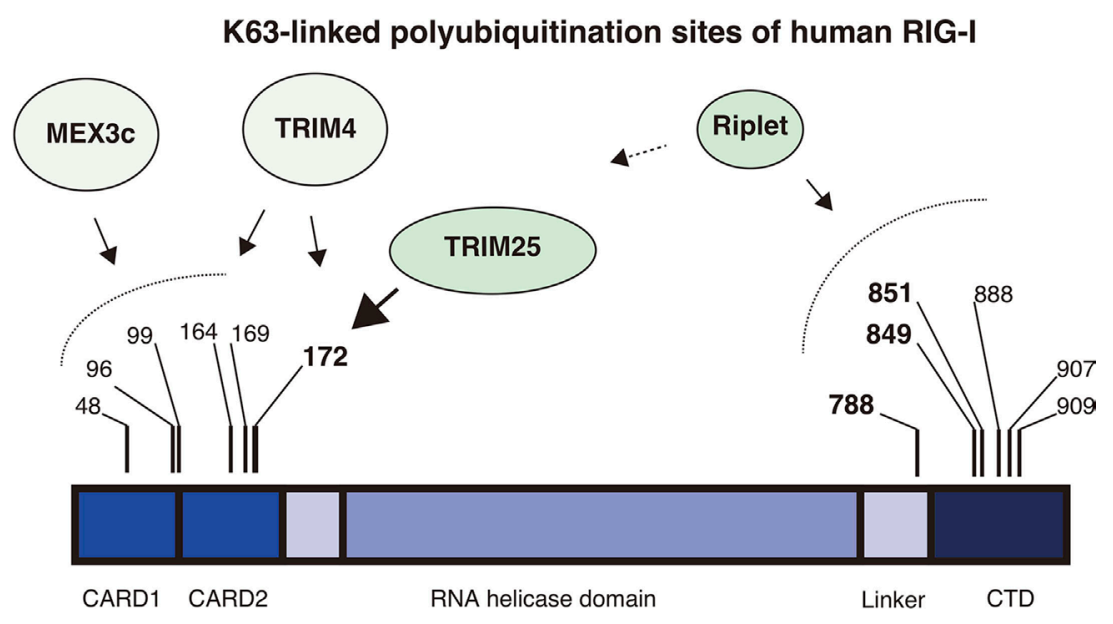

FIGURE 1 | K63-linked polyubiquitination sites of RIG-I. The RIG-I protein harbors K63-linked polyubiquitination at multiple sites. The protein comprises the $\mathrm{N}$-terminal 2CARDs, the RNA helicase domain, and the C-terminal domain (CTD). The four ubiquitin ligases, Riplet, TRIM25, MEX3C, and TRIM4 are involved in the ubiquitination. Mass spectrometry analyses showed that Lys 172 of the 2CARDs carries K63-linked polyubiquitination. TRIM25 mediates K63-linked polyubiquitination at Lys 172 of the 2CARDs or deliver unanchored K63-linked polyubiquitin chains to it. MEX3C mediates K63-linked polyubiquitination at Lys 48, 99, and 169. TRIM4 mediates it at Lys 164 and 172. Shi et al. reported that Lys 96 of the 2CARDs carried K63-linked polyubiquitin chain and were required for RIG-I activation. By contrast, the Riplet ubiquitin ligase mediates K63-linked polyubiquitination of the CTD and the linker region between the CTD and the RNA helicase domain. Lys 788, 849, and 851 are important for RIG-I activation, but other Lys residues, including Lys 888, 907, and 909, also harbor ubiquitination.

vesicular stomatitis virus (VSV) infections. In addition, Riplet KO mice were susceptible to VSV infection compared with wildtype mice (35). These observations indicate that Riplet-mediated polyubiquitination of RIG-I is essential for RIG-I activation during viral infection in vivo.

An RIG-I C-terminal fragment (735-925 aa region), which includes the linker region and the CTD, suppresses the 2CARD activation (14), and Kageyama et al. reported that the linker region (746-801 aa) was responsible for the auto-suppression (18). Riplet targets the K788 in the linker region. Therefore, it is possible that the ubiquitination of the linker region disrupts the auto-suppression. The structure analysis revealed that K849, $\mathrm{K} 851, \mathrm{~K} 858$, and $\mathrm{K} 888$ of the CTD bind to $5^{\prime}$ triphosphate of dsRNA ends (36). The K849, K851, and K888 of the CTD are targeted by Riplet. Binding of the CTD to $5^{\prime}$ triphosphate of dsRNA was reported to induce conformational change of the RIG-I protein (16). Although several RIG-I molecules assemble along dsRNA, only one RIG-I molecule binds the $5^{\prime}$ triphosphate at the dsRNA end (19) (Figure 2A). Therefore, Riplet could access the K849, K851, and K888 of the CTDs of the RIG-I molecules associating with dsRNA (but not the end of dsRNA) to induce conformational change of RIG-I. These $\mathrm{K}$ residues are located at the edge of the CTD basic cleft, which is an RNA-binding site (16). Further studies are required to reveal underlying mechanism of Riplet-mediated RIG-I activation.

\section{SEQUENTIAL UBIQUITINATION MODEL}

Despite the identification of four ubiquitin ligases, we found that knockout of Riplet alone could abolish the polyubiquitination of the endogenous RIG-I protein $(24,35)$. Recently, Shi et al. also reported that knockout of Riplet is sufficient to abolish the polyubiquitination of RIG-I and, therefore, claimed that Riplet is a primary ubiquitin ligase and mediates K63-linked polyubiquitination of the 2CARDs (34). However, their model appears to be contradict to previous papers showing that TRIM25 plays a crucial role in RIG-I activation.

Previously, we have postulated a sequential ubiquitination model that Riplet-mediated polyubiquitination of RIG-I $\mathrm{C}$-terminal region is a prerequisite for the polyubiquitination of the 2CARDs (Figure 2) (24). This model could explain the apparent discrepancy in the literature, because due to the initial failure to polyubiquitinate the $\mathrm{C}$-terminal region, this would obstruct the subsequent polyubiquitination of the 2CARDs by other ubiquitin ligases. This indicates that knockout of Riplet alone is sufficient to abolish the polyubiquitination of the CTD, the linker region, and the 2 CARDs. In a previous study, we have shown that Riplet promotes the binding of TRIM25 to RIG-I (24). This observation supports the sequential model. It is expected that Riplet-mediated polyubiquitination leads to the release of auto-suppression and/or conformational change of RIG-I, which would allow the access of TRIM 25 to the 2CARDs and/or promote RIG-I assembly along dsRNA (Figure 2B). Considering that higher-order oligomerization of TRIM25 with the 2CARDs is required to induce TRIM25-mediated polyubiquitination (31), it is not surprising that Riplet-mediated C-terminal ubiquitination is a prerequisite for the second ubiquitination by TRIM25 (Figure 2B).

MEX3C or TRIM4 might compensate for the defect of TRIM25 under certain experimental conditions, because these two ubiquitin ligases target the 2CARDs in a similar way (Figure 1). Although we failed to detect an interaction between Riplet and the 2CARDs fragment, other groups have reported that Riplet bound to the 2CARDs fragment and was involved in the K63-linked 


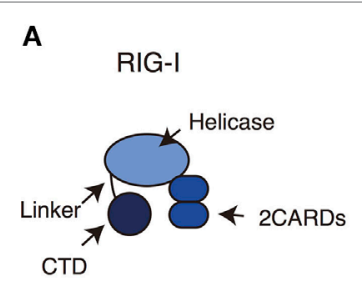

B
RIG-I molecules assembe along dsRNA

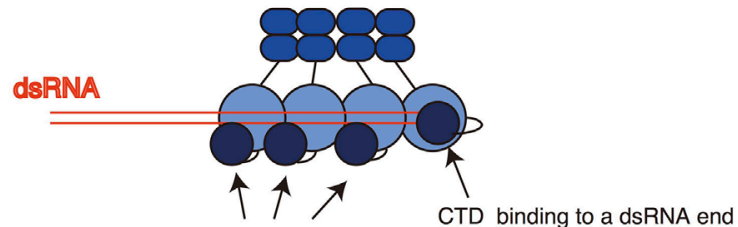

CTDs binding to dsRNA but not a dsRNA end

RIG-I

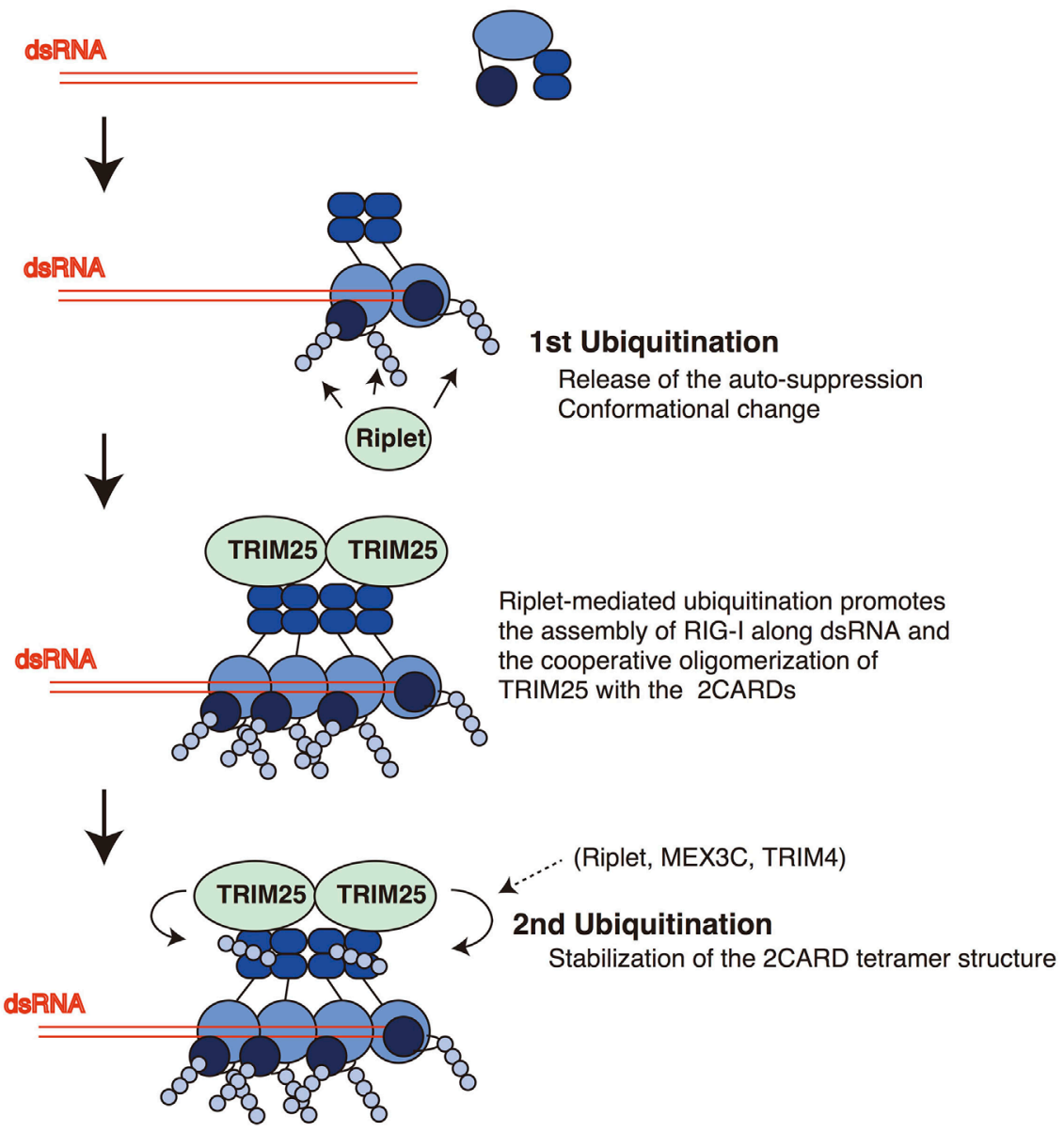

FIGURE 2 Sequential ubiquitination model for RIG-I. (A) The RIG-I proteins assemble along double-stranded RNA (dsRNA). The C-terminal domain (CTD) recognizes 5' triphosphate at the end of dsRNA. One CTD is required for the recognition of 5' triphosphate, whereas other CTDs of RIG-I molecules that assemble along dsRNA do not bind the end of dsRNA. (B) Riplet mediates K63-linked polyubiquitination of the linker region of RIG-I associated with dsRNA. In addition, Riplet can ubiquitinate the CTDs that assemble along dsRNA but not at the end of dsRNA. The linker region is responsible for the auto-suppression of the 2CARDs, and the CTD activation promotes the conformational change of the RIG-I protein. Riplet-mediated polyubiquitination of the C-terminal region (first ubiquitination) is expected to promote the release of the auto-suppression and/or the assembly of RIG-I along dsRNA via conformational change, leading to the binding of TRIM25 to the 2CARDs. Subsequently, cooperative oligomerization of TRIM25 with the 2CARDs induces the TRIM25-mediated polymerization of ubiquitin (second ubiquitination) required for stabilizing the 2CARD tetramer structure.

polyubiquitination of the 2CARDs $(34,37)$. These observations do not conflict with the sequential ubiquitination model because several ubiquitin ligases can compensate for the loss of TRIM25 in some conditions. We do not exclude the possibility that Riplet is not only involved in the primary ubiquitination of the CTD and the linker region but also the secondary ubiquitination of the 2CARDs (Figure 2).

\section{VIRAL TARGETING OF UBIQUITIN LIGASES}

Type I IFN exhibits a strong antiviral effect, and hence several viruses have evolved to suppress the type I IFN production. HCV is a major cause of hepatocellular carcinoma and persistently infects hepatocytes over several decades without 
exclusion by the host immune system. A viral NS3-4A protease is required to cleave viral polypeptides and produce mature viral proteins; however, it is also important to suppress the host innate immune response. NS3-4A of HCV cleaves MAVS, which results in the release of MAVS from mitochondria (12). Several reports have shown that released MAVS protein fails to trigger signaling to induce type I IFN production (9). Accordingly, NS3-4A-mediated cleavage of MAVS abrogates RIG-I-mediated type I IFN production. NS3-4A protein also targets the Riplet protein. The RING finger domain is a catalytic domain of the Riplet protein, and viral NS3-4A protease cleaves the domain and destabilizes the protein (24). NS1 protein of influenza A virus also has the ability to suppress type I IFN production (38). Although several mechanisms have been postulated, Gack et al. reported that viral NS1 bound to TRIM25 and inhibited TRIM25-mediated polyubiquitination of RIG-I (39). In further study, they reported that NS1 protein also targeted the Riplet protein and inhibited RIG-I polyubiquitination (40). Severe acute respiratory syndrome coronavirus (SARS-CoV) also interferes TRIM25 function (41). The nucleocapsid protein of SARS-CoV physically interacted with TRIM25 and inhibited the binding of TRIM25 to RIG-I, resulting in the attenuation of RIG-I-signaling (41). These data imply that viruses obtained the ability to suppress the ubiquitin ligases to escape innate immune responses. Conversely, these data indicate the importance of the two ubiquitin ligases, TRIM25 and Riplet, for the antiviral innate immune response.

\section{PERSPECTIVES}

TRIM25 is also called EFP, and it has been shown that TRIM25/ EFP mediates the polyubiquitination of $14-3-3 \sigma$ and promotes its proteolysis to suppress the growth of breast tumor cells (42). Although other targets of Riplet have not been reported, it was shown that mutations on human Riplet genes (also called RNF135)

\section{REFERENCES}

1. Loo YM, Gale M Jr. Immune signaling by RIG-I-like receptors. Immunity (2011) 34:680-92. doi:10.1016/j.immuni.2011.05.003

2. Kawai T, Akira S. Toll-like receptors and their crosstalk with other innate receptors in infection and immunity. Immunity (2011) 34:637-50. doi:10.1016/j. immuni.2011.05.006

3. Goubau D, Schlee M, Deddouche S, Pruijssers AJ, Zillinger T, Goldeck M, et al. Antiviral immunity via RIG-I-mediated recognition of RNA bearing 5'-diphosphates. Nature (2014) 514:372-5. doi:10.1038/nature13590

4. Hornung V, Ellegast J, Kim S, Brzozka K, Jung A, Kato H, et al. 5'-Triphosphate RNA is the ligand for RIG-I. Science (2006) 314:994-7. doi:10.1126/ science. 1132505

5. Yoneyama M, Kikuchi M, Natsukawa T, Shinobu N, Imaizumi T, Miyagishi M, et al. The RNA helicase RIG-I has an essential function in double-stranded RNA-induced innate antiviral responses. Nat Immunol (2004) 5:730-7. doi:10.1038/ni1087

6. Kato H, Takeuchi O, Mikamo-Satoh E, Hirai R, Kawai T, Matsushita K, et al. Length-dependent recognition of double-stranded ribonucleic acids by retinoic acid-inducible gene-I and melanoma differentiation-associated gene 5. J Exp Med (2008) 205:1601-10. doi:10.1084/jem.20080091

7. Loo YM, Fornek J, Crochet N, Bajwa G, Perwitasari O, Martinez-Sobrido L, et al. Distinct RIG-I and MDA5 signaling by RNA viruses in innate immunity. J Virol (2008) 82:335-45. doi:10.1128/JVI.01080-07 are linked to learning disabilities and several neuropsychiatric disorders $(43,44)$. Thus, it is expected that Riplet targets the proteins involved in these conditions. There are several reports that Riplet and TRIM25 are involved in tumorigenesis $(42,45,46)$. As several viruses have the ability to abrogate the described ubiquitin ligases, it is expected that viral protein-mediated inhibition of the ubiquitin ligases affects both innate immunity and other phenomena, such as virus-induced tumorigenesis and neuropsychiatric disorders.

There are two protein families related to RIG-I called RIG-Ilike receptors (RLRs). LGP2 is an RLR, and the CTD structure of the protein is similar to that of RIG-I (14). Initial studies reported that LGP2 is a negative regulator for RIG-I signaling $(15,47)$. However, knockout and biochemical studies have revealed that LGP2 functions as a positive regulator of the RIG-I pathway $(48,49)$. LGP2 is also expressed in CD8 ${ }^{+} \mathrm{T}$ cells and is required for $\mathrm{CD}^{+} \mathrm{T}$ cell proliferation (50). It remains unclear whether LGP2 carries a ubiquitin chain. Considering the conservation of the CTD between RIG-I and LGP2, it is possible that Riplet also targets the CTD of LGP2 and affects LGP2-mediated RIG-I activation and $\mathrm{CD} 8^{+} \mathrm{T}$ cell proliferation. Further studies are required to fully elucidate the role of the ubiquitin ligases in the antiviral immune response.

\section{AUTHOR CONTRIBUTIONS}

$\mathrm{HO}$ and MO wrote the manuscript. TK and YF helped the discussion.

\section{ACKNOWLEDGMENTS}

The authors thank Dr. T. Seya and Dr. M. Matsumoto for their helpful discussion. This work was supported in part by Grantsin-Aid from Ministry of Education, Science, and Culture, and PRESTO JST.
8. Kato H, Takeuchi O, Sato S, Yoneyama M, Yamamoto M, Matsui K, et al Differential roles of MDA5 and RIG-I helicases in the recognition of RNA viruses. Nature (2006) 441:101-5. doi:10.1038/nature04734

9. Seth RB, Sun L, Ea CK, Chen ZJ. Identification and characterization of MAVS, a mitochondrial antiviral signaling protein that activates NF-kappaB and IRF 3. Cell (2005) 122:669-82. doi:10.1016/j.cell.2005.08.012

10. Kawai T, Takahashi K, Sato S, Coban C, Kumar H, Kato H, et al. IPS-1, an adaptor triggering RIG-I- and Mda5-mediated type I interferon induction. Nat Immunol (2005) 6:981-8. doi:10.1038/ni1243

11. Xu LG, Wang YY, Han KJ, Li LY, Zhai Z, Shu HB. VISA is an adapter protein required for virus-triggered IFN-beta signaling. Mol Cell (2005) 19:727-40. doi:10.1016/j.molcel.2005.08.014

12. Meylan E, Curran J, Hofmann K, Moradpour D, Binder M, Bartenschlager R, et al. Cardif is an adaptor protein in the RIG-I antiviral pathway and is targeted by hepatitis C virus. Nature (2005) 437:1167-72. doi:10.1038/nature04193

13. Hou F, Sun L, Zheng H, Skaug B, Jiang QX, Chen ZJ. MAVS forms functional prion-like aggregates to activate and propagate antiviral innate immune response. Cell (2011) 146:448-61. doi:10.1016/j.cell.2011.06.041

14. Saito T, Hirai R, Loo YM, Owen D, Johnson CL, Sinha SC, et al. Regulation of innate antiviral defenses through a shared repressor domain in RIG-I and LGP2. Proc Natl Acad Sci U S A (2007) 104:582-7. doi:10.1073/ pnas.0606699104

15. Yoneyama M, Kikuchi M, Matsumoto K, Imaizumi T, Miyagishi M, Taira K, et al. Shared and unique functions of the DExD/H-box helicases RIG-I, 
MDA5, and LGP2 in antiviral innate immunity. J Immunol (2005) 175:2851-8. doi:10.4049/jimmunol.175.5.2851

16. Takahasi K, Yoneyama M, Nishihori T, Hirai R, Kumeta H, Narita R, et al. Nonself RNA-sensing mechanism of RIG-I helicase and activation of antiviral immune responses. Mol Cell (2008) 29:428-40. doi:10.1016/j. molcel.2007.11.028

17. Cui S, Eisenacher K, Kirchhofer A, Brzozka K, Lammens A, Lammens K, et al. The C-terminal regulatory domain is the RNA 5'-triphosphate sensor of RIG-I. Mol Cell (2008) 29:169-79. doi:10.1016/j.molcel.2007.10.032

18. Kageyama M, Takahasi K, Narita R, Hirai R, Yoneyama M, Kato H, et al. 55 Amino acid linker between helicase and carboxyl terminal domains of RIG-I functions as a critical repression domain and determines inter-domain conformation. Biochem Biophys Res Commun (2011) 415:75-81. doi:10.1016/j. bbrc.2011.10.015

19. Peisley A, Wu B, Yao H, Walz T, Hur S. RIG-I forms signaling-competent filaments in an ATP-dependent, ubiquitin-independent manner. Mol Cell (2013) 51:573-83. doi:10.1016/j.molcel.2013.07.024

20. Wu B, Peisley A, Richards C, Yao H, Zeng X, Lin C, et al. Structural basis for dsRNA recognition, filament formation, and antiviral signal activation by MDA5. Cell (2013) 152:276-89. doi:10.1016/j.cell.2012.11.048

21. Peisley A, Wu B, Xu H, Chen ZJ, Hur S. Structural basis for ubiquitin-mediated antiviral signal activation by RIG-I. Nature (2014) 509:110-4. doi:10.1038/ nature 13140

22. Gack MU, Shin YC, Joo CH, Urano T, Liang C, Sun L, et al. TRIM25 RINGfinger E3 ubiquitin ligase is essential for RIG-I-mediated antiviral activity. Nature (2007) 446:916-20. doi:10.1038/nature05732

23. Zeng W, Sun L, Jiang X, Chen X, Hou F, Adhikari A, et al. Reconstitution of the RIG-I pathway reveals a signaling role of unanchored polyubiquitin chains in innate immunity. Cell (2010) 141:315-30. doi:10.1016/j.cell.2010.03.029

24. Oshiumi H, Miyashita M, Matsumoto M, Seya T. A distinct role of Ripletmediated K63-linked polyubiquitination of the RIG-I repressor domain in human antiviral innate immune responses. PLoS Pathog (2013) 9:e1003533. doi:10.1371/journal.ppat.1003533

25. Arnaud N, Dabo S, Akazawa D, Fukasawa M, Shinkai-Ouchi F, Hugon J, et al. Hepatitis $\mathrm{C}$ virus reveals a novel early control in acute immune response. PLoS Pathog (2011) 7:e1002289. doi:10.1371/journal.ppat.1002289

26. Chiang C, Gack MU. Post-translational control of intracellular pathogen sensing pathways. Trends Immunol (2017) 38:39-52. doi:10.1016/j.it. 2016.10.008

27. Chan YK, Gack MU. Viral evasion of intracellular DNA and RNA sensing. Nat Rev Microbiol (2016) 14:360-73. doi:10.1038/nrmicro.2016.45

28. Oshiumi H, Matsumoto M, Hatakeyama S, Seya T. Riplet/RNF135, a RING finger protein, ubiquitinates RIG-I to promote interferon-beta induction during the early phase of viral infection. J Biol Chem (2009) 284:807-17. doi:10.1074/jbc.M804259200

29. Kuniyoshi K, Takeuchi O, Pandey S, Satoh T, Iwasaki H, Akira S, et al. Pivotal role of RNA-binding E3 ubiquitin ligase MEX3C in RIG-I-mediated antiviral innate immunity. Proc Natl Acad Sci U S A (2014) 111:5646-51. doi:10.1073/pnas.1401674111

30. Yan J, Li Q, Mao AP, Hu MM, Shu HB. TRIM4 modulates type I interferon induction and cellular antiviral response by targeting RIG-I for K63-linked ubiquitination. J Mol Cell Biol (2014) 6:154-63. doi:10.1093/jmcb/mju005

31. Sanchez JG, Chiang JJ, Sparrer KMJ, Alam SL, Chi M, Roganowicz MD, et al. Mechanism of TRIM25 catalytic activation in the antiviral RIG-I pathway. Cell Rep (2016) 16:1315-25. doi:10.1016/j.celrep.2016.06.070

32. Oshiumi H, Matsumoto M, Seya T. Ubiquitin-mediated modulation of the cytoplasmic viral RNA sensor RIG-I. J Biochem (2012) 151:5-11. doi:10.1093/ $\mathrm{jb} / \mathrm{mvr} 111$

33. Shigemoto T, Kageyama M, Hirai R, Zheng J, Yoneyama M, Fujita T. Identification of loss of function mutations in human genes encoding RIG-I and MDA5: implications for resistance to type I diabetes. J Biol Chem (2009) 284:13348-54. doi:10.1074/jbc.M809449200

34. Shi Y, Yuan B, Zhu W, Zhang R, Li L, Hao X, et al. Ube2D3 and Ube2N are essential for RIG-I-mediated MAVS aggregation in antiviral innate immunity. Nat Commun (2017) 8:15138. doi:10.1038/ncomms15138
35. Oshiumi H, Miyashita M, Inoue N, Okabe M, Matsumoto M, Seya T. The ubiquitin ligase Riplet is essential for RIG-I-dependent innate immune responses to RNA virus infection. Cell Host Microbe (2010) 8:496-509. doi:10.1016/j.chom.2010.11.008

36. Luo D, Kohlway A, Vela A, Pyle AM. Visualizing the determinants of viral RNA recognition by innate immune sensor RIG-I. Structure (2012) 20:1983-8. doi:10.1016/j.str.2012.08.029

37. Gao D, Yang YK, Wang RP, Zhou X, Diao FC, Li MD, et al. REUL is a novel E3 ubiquitin ligase and stimulator of retinoic-acid-inducible gene-I. PLoS One (2009) 4:e5760. doi:10.1371/journal.pone.0005760

38. Diebold SS, Montoya M, Unger H, Alexopoulou L, Roy P, Haswell LE, et al. Viral infection switches non-plasmacytoid dendritic cells into high interferon producers. Nature (2003) 424:324-8. doi:10.1038/nature01783

39. Gack MU, Albrecht RA, Urano T, Inn KS, Huang IC, Carnero E, et al. Influenza A virus NS1 targets the ubiquitin ligase TRIM25 to evade recognition by the host viral RNA sensor RIG-I. Cell Host Microbe (2009) 5:439-49. doi:10.1016/j.chom.2009.04.006

40. Rajsbaum R, Albrecht RA, Wang MK, Maharaj NP, Versteeg GA, Nistal-Villan E, et al. Species-specific inhibition of RIG-I ubiquitination and IFN induction by the influenza A virus NS1 protein. PLoS Pathog (2012) 8:e1003059. doi:10.1371/journal.ppat.1003059

41. Hu Y, Li W, Gao T, Cui Y, Jin Y, Li P, et al. The severe acute respiratory syndrome coronavirus nucleocapsid inhibits type I interferon production by interfering with TRIM25-mediated RIG-I ubiquitination. J Virol (2017) 91:e02143-16. doi:10.1128/JVI.02143-16

42. Urano T, Saito T, Tsukui T, Fujita M, Hosoi T, Muramatsu M, et al. Efp targets 14-3-3 sigma for proteolysis and promotes breast tumour growth. Nature (2002) 417:871-5. doi:10.1038/nature00826

43. Douglas J, Cilliers D, Coleman K, Tatton-Brown K, Barker K, Bernhard B, et al. Mutations in RNF135, a gene within the NF1 microdeletion region, cause phenotypic abnormalities including overgrowth. Nat Genet (2007) 39:963-5. doi:10.1038/ng2083

44. Tastet J, Decalonne L, Marouillat S, Malvy J, Thepault RA, Toutain A, et al. Mutation screening of the ubiquitin ligase gene RNF135 in French patients with autism. Psychiatr Genet (2015) 25:263-7. doi:10.1097/YPG.0000000000000100

45. Liu Y, Wang F, Liu Y, Yao Y, Lv X, Dong B, et al. RNF135, RING finger protein, promotes the proliferation of human glioblastoma cells in vivo and in vitro via the ERK pathway. Sci Rep (2016) 6:20642. doi:10.1038/srep20642

46. Jin J, Zhao L, Li Z. The E3 ubiquitin ligase RNF135 regulates the tumorigenesis activity of tongue cancer SCC25 cells. Cancer Med (2016) 5:3140-6. doi:10.1002/cam4.832

47. Venkataraman T, Valdes M, Elsby R, Kakuta S, Caceres G, Saijo S, et al. Loss of DExD/H box RNA helicase LGP2 manifests disparate antiviral responses. J Immunol (2007) 178:6444-55. doi:10.4049/jimmunol.178.10.6444

48. Satoh T, Kato H, Kumagai $\mathrm{Y}$, Yoneyama M, Sato $\mathrm{S}$, Matsushita $\mathrm{K}$, et al. LGP2 is a positive regulator of RIG-I- and MDA5-mediated antiviral responses. Proc Natl Acad Sci U S A (2010) 107:1512-7. doi:10.1073/pnas.0912986107

49. Bruns AM, Leser GP, Lamb RA, Horvath CM. The innate immune sensor LGP2 activates antiviral signaling by regulating MDA5-RNA interaction and filament assembly. Mol Cell (2014) 55:771-81. doi:10.1016/j. molcel.2014.07.003

50. Suthar MS, Ramos HJ, Brassil MM, Netland J, Chappell CP, Blahnik G, et al. The RIG-I-like receptor LGP2 controls CD8(+) T cell survival and fitness. Immunity (2012) 37:235-48. doi:10.1016/j.immuni.2012.07.004

Conflict of Interest Statement: The authors declare that the research was conducted in the absence of any commercial or financial relationships that could be construed as a potential conflict of interest.

Copyright $\odot 2018$ Okamoto, Kouwaki, Fukushima and Oshiumi. This is an open-access article distributed under the terms of the Creative Commons Attribution License (CC BY). The use, distribution or reproduction in other forums is permitted, provided the original author(s) or licensor are credited and that the original publication in this journal is cited, in accordance with accepted academic practice. No use, distribution or reproduction is permitted which does not comply with these terms. 